\title{
Proton NMR Observation of Phenylalanine and an Aromatic Metabolite in the Rabbit Brain in Vivo
}

\author{
M. J. AVISON, N. HERSCHKOWITZ, E. J. NOVOTNY, O. A. C. PETROFF, D. L. ROTHMAN, \\ J. P. COLOMBO, C. BACHMANN, R. G. SHULMAN, AND J. W. PRICHARD \\ Yale University, New Haven, Connecticut 06510 [M.J.A., E.J.N., O.A.C.P., D.L.R., R.G.S., J.W.P.], and \\ University of Bern, Switzerland [N.H., J.P.C., C.B.]
}

\begin{abstract}
H}$ nuclear magnetic resonance (NMR) was used to detect directly the signal from the aromatic protons of phenylaline (phe) in the brains of rabbits made hyperphenylalaninemic by administration of a diet high in phe and containing $0.4 \% \alpha$-methylphenylalanine. In addition to those resonances found in the region between 6.5 and 8.5 ppm in the ${ }^{1} \mathrm{H}$ NMR spectra of control rabbits, a resonance centered at $7.37 \mathrm{ppm}$ was observed in the spectra obtained from the brains of hyperphenylalaninemic rabbits in vivo or in situ postmortem. The chemical shift of this additional resonance was that expected for protons of the phenyl ring of phe. Its intensity correlated well with measurements of brain phe levels made on postmortem samples by amino acid analyzer. Both of these measurements correlated poorly with amino acid analyzer measurements of serum phe, especially at high values of the latter. High-resolution ${ }^{1} H$ NMR spectra of the brain extracts showed that in most animals an unidentified aromatic compound, possibly $\gamma$ glutamyl-phe, was present in addition to phe. This study demonstrates the feasibility of measuring the concentration of brain phenyl and its metabolites noninvasively by ${ }^{1} \mathrm{H}$ NMR. The method can be used for similar measurements in human brain. (Pediatr Res 27: 566-570, 1990)
\end{abstract}

\section{Abbreviations}

hyper-phe, hyperphenylalaninemia

NMR, nuclear magnetic resonance

phe, phenylalanine

[phe $]_{\text {aaa }}$, phenylalanine concentration determined by amino acid analyzer

phenyl, phenylalanine plus other small molecules with phenyl protons

PKU, phenylketonuria

$\mathrm{Cr}$, creatine + phosphocreatine.

Diseases resulting in hyper-phe are among the most common inborn errors of metabolism ( 1 in 14000 newborns in the United States) (1). The best known, PKU, results from a mutation in the long arm of chromosome 12 causing a defect in the hepatic enzyme phe-4-monooxygenase, which converts phe to tyrosine and is required for the hepatic catabolism of phe (2). In the absence of this enzyme, the serum level of phe rises from the

Received August 21, 1989; accepted February 12, 1990

Correspondence: Edward J. Novotny, M.D., Dept. of Neurology, Yale Medical School, 333 Cedar Street, New Haven, CT 06510.

Supported by grants from the United States Public Health Service (GM 30287 , NS 21708) and Swiss National Science Foundation (3.156.0.88). E. J. Novotny was supported in part by a National Research Service Award (NS 08252). normal value of $\sim 0.1 \mathrm{mM}$ to as high as $5 \mathrm{mM}$. The consequences of elevated serum phe for the developing brain of the newborn and fetus are catastrophic, resulting in severe mental retardation unless steps are taken to reduce serum phe to the normal range as soon as possible. This is achieved by placing PKU patients on a diet that contains only sufficient phe to meet dietary requirements. Dietary compliance is generally monitored by measuring phe in patients' serum by amino acid analyzer (serum [phe $]_{\mathrm{aaa}}$ ). In the first two decades that this disorder has been recognized and treated, most PKU patients returned to a normal diet in adolescence. Several recent reports indicate that, in some cases, return to a normal diet leads to both short- and long-term neuropsychologic deficits $(3,4)$. It has proved difficult to predict on the basis of serum [phe] $]_{\text {aaa }}$ which patients might be most at risk when they come off the low-phe diet. Because phe crosses the blood-brain barrier slowly, measurements of phe levels directly in the brain might provide additional insight into this problem, but no method for making such measurements has been available.

In vivo NMR has been used to measure brain metabolites noninvasively in animals (5) and man (6). Gadian et al. (7) used ${ }^{1} \mathrm{H}$ NMR spectroscopy in vivo to measure brain histidine in histidinemic mice, and suggested that similar methods might allow observation of elevated brain phe. In our study, we show that the total concentration of phe and another yet unidentified phenyl-containing compound-possibly $\tau$-glutamyl phe-can be measured in the brains of hyper-phe rabbits in vivo by ${ }^{1} \mathrm{H}$ NMR. This quantity, which we designate "[phenyl]," appears to be a measure of the same compounds detected in postmortem brain samples by amino acid analyzer (brain [phe] $]_{\mathrm{aaa}}$ ). The in vivo ${ }^{1} \mathrm{H}$ NMR methods can be adapted for use in humans.

\section{MATERIALS AND METHODS}

Hyper-phe was induced in female New Zealand White rabbits by feeding them a diet containing $5 \%$ phe and $0.4 \% \alpha$-methyl phe, an inhibitor of phe-4-monooxygenase, for 7-21 d. In most animals, this dietary regimen caused a large elevation of serum [phe $]_{\text {aaa }}$ (Table 1). On the day of the experiment, animals fasted for $5-8 \mathrm{~h}$ were anesthetized (4\% isoflurane), tracheostomized, paralyzed ( $3 \mathrm{mg} / \mathrm{kg}$ tubocurare, $2 \mathrm{mg} / \mathrm{kg}$ pancuronium) and pump ventilated with $50 \% \mathrm{NO}_{2} / 50 \% \mathrm{O}_{2}$. Four animals, including those with the highest serum [phe $]_{\text {aaa }}$, did not survive the surgery. Nonetheless, all animals were prepared for in situ determination of brain [phenyl] by ${ }^{1} \mathrm{H}$ NMR. The postmortem measurements are identified by the D prefix in Table 1 , whereas those animals for which the brain [phenyl] was determined in vivo constitute the A series. Serum samples were taken at the end of each experiment. Five age-matched animals fed a normal diet were also prepared in this fashion.

For NMR measurements, the animal was mounted in a holder, 
the scalp was retracted, and a $16-\mathrm{mm}$ diameter surface coil was placed $\sim 2 \mathrm{~mm}$ above the exposed skull, centered on the midline $8 \mathrm{~mm}$ posterior to the bregma. ${ }^{1} \mathrm{H}$ NMR spectra were obtained using a Bruker/Oxford Research Systems (Billerica, MA) Biospec I spectrometer equipped with a 4.7 Tesla horizontal magnet operating at $200.44 \mathrm{MHz}$ for protons.

A simple spin echo sequence $\left(\theta-\mathrm{t}_{\mathrm{e}} / 2-2 \theta-\mathrm{t}_{\mathrm{e}} / 2-\mathrm{AQ}\right)$ was used, with the $\theta$ and $2 \theta$ pulses being on-resonance-suppressed binomial $1 \overline{1}$ and $2 \overline{2}$ pulses (8), to achieve the required water suppression ( $t_{e}$ refers to total echo time; AQ refers to acquisition time). The phases of the $2 \theta$ pulse and the receiver were cycled using the EXORCYCLE scheme of Bodenhausen et al. (9). A value of 2 ms was used for $t_{e} / 2$ in all experiments to minimize the signal loss due to the relatively short $T_{2}\left(T_{2}=\right.$ transverse relaxation time) of the phenyl ring aromatic protons ( $40 \mathrm{~ms}$ in rabbit brain at 4.7 Tesla; Novotny EJ, Ogino T, Petroff OA, Prichard JW, Shulman RG, unpublished data). Spectra were collected using a spectral width of $5 \mathrm{kHz}$, and 2048 points. A repetition time of 2 $\mathrm{s}$ was used, and a sufficiently large signal-to-noise ratio was generally obtained with 256 averages. Convolution difference and Gaussian filtering were used before Fourier transformation to improve spectral resolution. All spectra were processed identically. Because the calculation of [phenyl] relies on accurate determination of the ratio of the phenyl and total creatine intensities in the in situ ${ }^{1} \mathrm{H}$ spectra, we verified that this ratio was not altered by the resolution enhancement. To do this, we broadened the free induction decay of a single water resonance by exponential multiplication to the linewidth of $\mathrm{Cr}$ or phenyl observed in situ. These broadened free induction decays were then filtered using the same processing parameters used in the in situ spectra. The intensity (I) ratios of these two resonances were the same before and after resolution enhancement, showing that the $\mathrm{I}_{\text {phenyl }} / \mathrm{I}_{\mathrm{Cr}}$ ratio was not changed by this procedure.

Intensities of in situ phenyl resonances were determined in two ways. The first consisted of subtracting an appropriately scaled normal spectrum from the hyper-phe spectrum to leave a

Table 1. Eleven hyper-phe animals studied by ${ }^{1} H$ NMR of brain in situ*

\begin{tabular}{|c|c|c|c|c|c|}
\hline \multirow[b]{3}{*}{$\begin{array}{c}\text { Experiment } \\
\text { no. }\end{array}$} & \multirow[b]{3}{*}{ Serum [phe] $]_{\text {aaa }}$} & \multicolumn{3}{|c|}{ Brain measurements } & \multirow[b]{3}{*}{$\begin{array}{l}\mathrm{X}^{\dagger} \\
(\%)\end{array}$} \\
\hline & & \multicolumn{2}{|c|}{ Extracts } & \multirow{2}{*}{$\begin{array}{c}\frac{\text { [phenyl] }}{{ }^{1} \mathrm{H}} \\
\text { NMR }\end{array}$} & \\
\hline & & $\begin{array}{l}\text { a.a.a. } \neq \\
(\mathrm{mM})\end{array}$ & $\begin{array}{c}\text { 'H } \\
\text { NMR\& }\end{array}$ & & \\
\hline A5 & 2.4 & 1.6 & 1.4 & 1.8 & $\|$ \\
\hline A 8 & 0.3 & 0.2 & $\|$ & $\|$ & $\|$ \\
\hline $\mathrm{A} 10$ & 5.1 & 2.3 & 1.6 & 2.0 & 0 \\
\hline Al1 & 0.2 & 0.1 & 0.1 & $\|$ & 48 \\
\hline A 14 & 2.2 & 2.1 & 1.4 & 1.5 & 60 \\
\hline A 15 & 3.6 & 2.4 & 1.7 & 1.7 & 50 \\
\hline A 16 & 3.3 & 2.6 & 2.2 & 2.5 & 74 \\
\hline D9 & 4.5 & 1.5 & 1.5 & 1.8 & 45 \\
\hline $\mathrm{D} 12$ & 12.3 & 2.8 & 3.3 & 2.4 & 34 \\
\hline D13 & 3.0 & 2.0 & 1.1 & 0.9 & 30 \\
\hline D17 & 8.4 & 2.3 & 1.2 & 2.1 & 19 \\
\hline Normals & 0.18 & 0.17 & $\|$ & $\|$ & $\|$ \\
\hline SEM & \pm 0.05 & \pm 0.05 & & & \\
\hline
\end{tabular}

* Seven animals were examined in vivo (A series) and four shortly after death (D series). Mean values for five normal animals appear at the bottom, and the percentage of compound $\mathrm{X}$ in the right column.

$\dagger$ Determined by assuming that the two upfield peaks of $X$ (see Fig. 3) correspond to the same mol \% of $X$ as the two upfield peaks of the phe multiplet do for phe.

$\ddagger$ Amino acid analyzer.

$\S$ Concentration represents the sum of the brain phe and compound $\mathrm{X}$ concentrations.

|| Below level of detection.

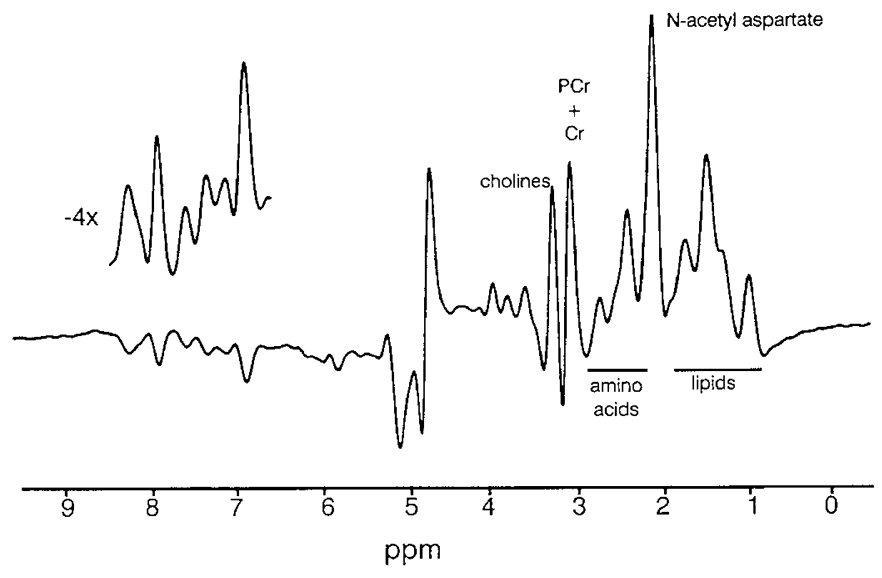

Fig. 1. Proton spectrum from living rabbit brain. ${ }^{1} \mathrm{H}$ NMR spectrum of normal rabbit brain obtained in vivo at 4.7 Tesla $(200.44 \mathrm{MHz})$. A semiselective spin echo sequence ( $1 \overline{1}-2 \overline{2}$ on-resonance suppressed) was used to suppress the water resonance at $4.73 \mathrm{ppm}$. The excitation maximum of the semiselective pulses was set at $7.37 \mathrm{ppm}$. The total echo time was $5 \mathrm{~ms}$. The spectrum was the sum of 256 transients collected with a $5 \mathrm{kHz}$ spectral width and 2048 data points. The spectrum was given a profile correction (Bruker DISNMR processing parameters: GB 200, DC 2) to remove any underlying broad resonances, and Gaussian resolution enhancement $(\mathrm{GB}=0.06 ; \mathrm{LB}=-20)$. Note that there is a phase reversal about the carrier frequency at $4.73 \mathrm{ppm}$, caused by the semiselective pulse. This causes the resonances downfield of water $(>4.73$ ppm) to be inverted with respect to the upfield peaks. The downfield region of interest is plotted, expanded, and inverted (segment labeled "-4X").

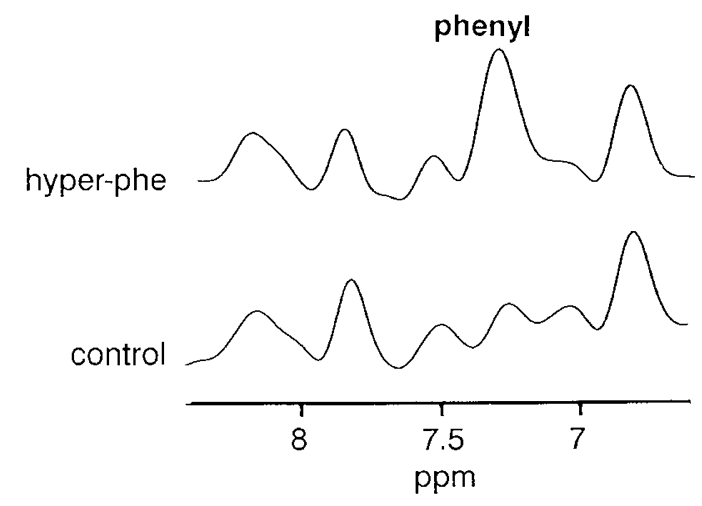

Fig. 2. Comparison of brain spectra from normal and hyper-phe rabbits. In vivo 'H NMR spectra of the downfield region from a control and a hyper-phe (experiment no A10). Note the additional peak at 7.37 ppm from the aromatic protons of phenyl. Spectral acquisition and processing conditions were as for Figure 1.

single peak at $7.37 \mathrm{ppm}$, whose area was then integrated with Bruker (Billerica, MA) DISNMR processing software and expressed as a ratio to the integrated area of the total creatine resonance. The second way was to define the areas of the phenyl and total creatine resonances in plotted spectra from hyper-phe animals as the portion of the resonance above a straight line between the points of inflection on either side of peaks with maxima at 7.37 or $3.03 \mathrm{ppm}$. These areas were measured with a digitizing tablet and commercially available software (SigmaScan, Jandel, Corte Madera, CA). In both methods, the phenyl/ total creatine area ratios were corrected for differences between the two resonances in nutation angle arising from the binomial pulses, saturation effects, $\mathrm{T}_{2} \mathrm{~S}$, and number of protons per molecule. There was a significant difference between brain [phenyl] values calculated by the two methods, but because there was less scatter in repeated measurements with the second method, it was used for further analysis. The equation used to convert the 

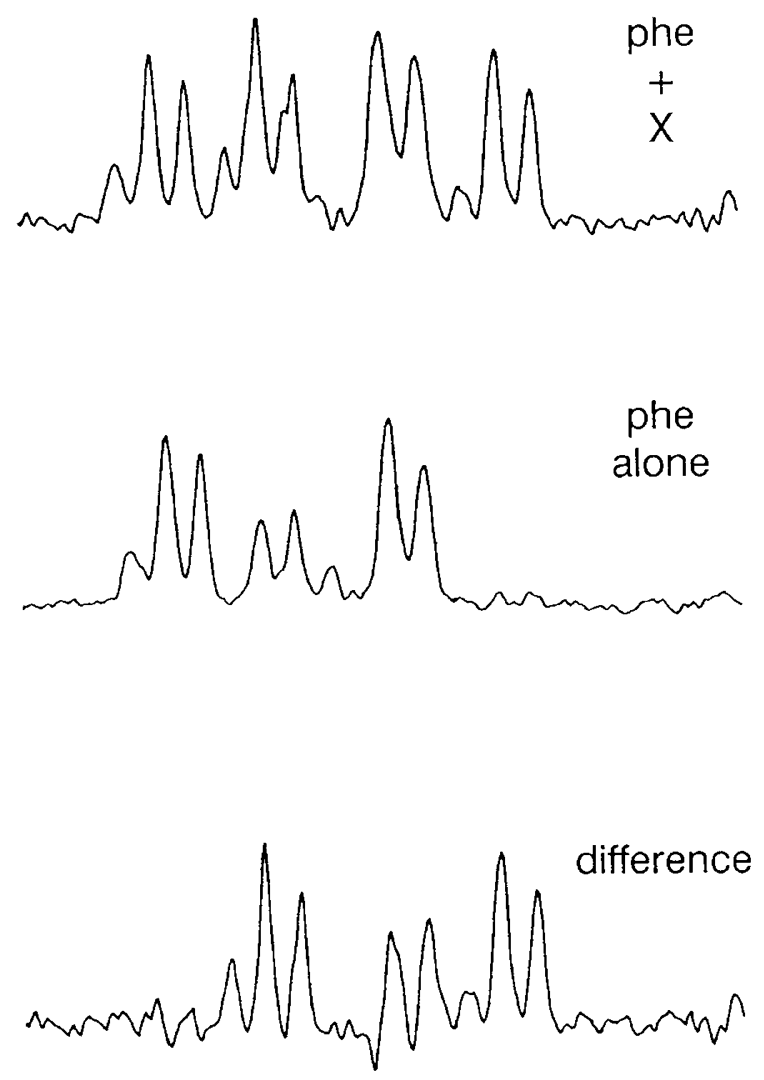

$\begin{array}{lllll}7.45 & 7.40 & 7.35 & 7.30 & 7.25\end{array}$

Fig. 3. Compound "X." In all but one animal, $500 \mathrm{MHz}{ }^{1} \mathrm{H}$ NMR spectra of brain extracts from hyper-phe rabbits contained an unidentified aromatic multiplet $(\mathrm{X})$ centered upfield of $7.37 \mathrm{ppm}$. The top spectrum shows an extract from a brain that contains almost equal amounts of phe and $\mathrm{X}$, whereas the middle spectrum shows an extract in which only phe is present. The bottom spectrum is the difference between the other two, showing the spectrum of $\mathrm{X}$ alone. Each spectrum consisted of the sum of 160 transients, and was given a Lorentzian to Gaussian conversion to improve spectral resolution (Bruker DISNMR parameters: GB $0.05, \mathrm{LB}-1$ ).

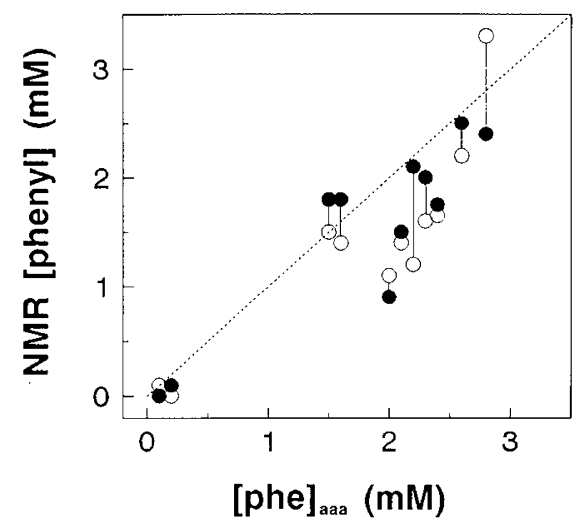

Fig. 4. Comparison of in vivo and in vitro measurements. Plot of brain [phenyl] determined by ${ }^{1} \mathrm{H}$ NMR in situ (filled circles) and in postmortem extracts (open circles), vs brain [phe] $]_{\text {aaa. }}$ The dotted line is the line of identity. Extract and in situ NMR values are linked by vertical bars.

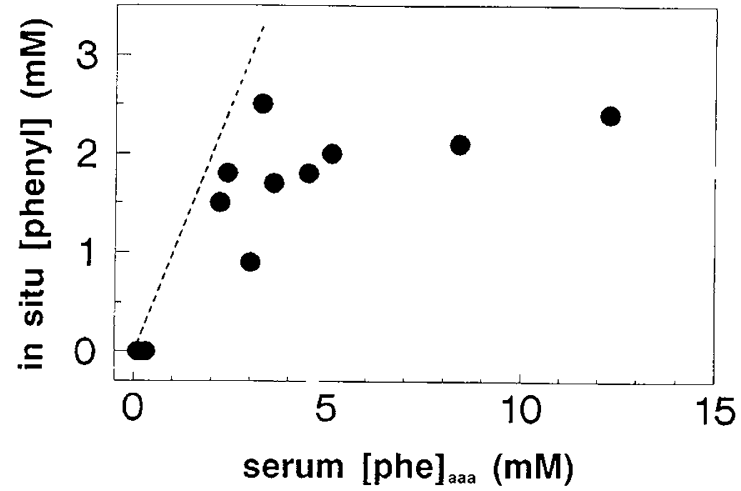

Fig. 5. Comparison of brain and serum measurements. Plot of brain [phenyl] determined in situ by 'H NMR vs serum [phe] ${ }_{\text {aaa. }}$.

intensity of the resonance from the aromatic protons of phenyl $\left(I_{\text {phenyl }}\right)$ to [phenyl] was derived from the expression for the ratio of the intensities of the phenyl and creatine resonances when the semiselective pulse is optimized for each in turn. Thus

$$
\frac{I_{\text {phenyl }}}{I_{C r}}=\frac{[\text { phenyl] }}{C r} \times \frac{n_{\text {phenyl }}}{n_{C r}} \times \frac{\exp \left(-t_{e} / T_{2(\text { phenyl })}\right)}{\exp \left(-t_{e} / T_{2(C r)}\right)} \times \frac{S F_{\text {phenyl }}}{S F_{C r}}
$$

where $\mathrm{I}_{\text {phenyl }}$ and $\mathrm{I}_{\mathrm{Cr}}$ are the intensities of the optimized resonances of the aromatic protons of phenyl at $7.37 \mathrm{ppm}$ and the methyl protons of total creatine at $3.03 \mathrm{ppm}, \mathrm{n}_{\text {phenyl }}$ and $\mathrm{n}_{\mathrm{Cr}}$ are the numbers of protons giving rise to each resonance, and $S_{F}$ phenyl and $\mathrm{SF}_{\mathrm{Cr}}$ are the empirically determined saturation factors for each resonance under the pulsing conditions used. This expression can be recast as

[phenyl] $=\frac{\mathrm{I}_{\text {phenyl }}}{\mathrm{I}_{\mathrm{Cr}}} \times \mathrm{CR} \times \frac{\mathrm{n}_{\mathrm{Cr}}}{\mathrm{n}_{\text {phenyl }}} \times \frac{\exp \left(-\mathrm{t}_{\mathrm{e}} / \mathrm{T}_{2(\mathrm{Cr})}\right)}{\exp \left(-\mathrm{t}_{\mathrm{e}} / \mathrm{T}_{2(\text { phenyl })}\right)} \times \frac{\mathrm{SF}_{\mathrm{Cr}}}{\mathrm{SF}_{\text {phenyl }}}$

where $\mathrm{T}_{2(\mathrm{Cr})}=140 \mathrm{~ms}, \mathrm{~T}_{2 \text { (phenyl) }}=38 \mathrm{~ms}, \mathrm{n}_{\mathrm{Cr}}=3$, and $\mathrm{n}_{\text {phenyl }}=$ 5 . The $T_{1}$ (longitudinal relaxation time) of the creatine resonance measured in vivo by inversion recovery at 4.7 Tesla was $1.5 \mathrm{~ms}$ (Novotny EJ, Ogino T, Petroff OA, Prichard JW, Shulman RG, unpublished data) and the saturation factors for the creatine and phenyl resonances determined by obtaining fully relaxed spectra (repetition time $10 \mathrm{~s}$ ) were, respectively, 1.17 and 1.16. $\mathrm{Cr}$ was assumed to represent a concentration of $9 \mathrm{mM}$ (12).

Once in situ phenyl measurements were complete, the seven living animals were killed by $\mathrm{KCl}$ injection; the phenyl resonance present in five of them did not change. Brains were removed from all animals and frozen for subsequent extraction of small metabolites. Extracts for chromatographic amino acid analysis were prepared using the method of Bachmann and Colombo (10); extracts for ${ }^{1} \mathrm{H}$ NMR were prepared according to the method of Behar et al. (11). Brain [phe] $]_{\text {aaa }}$ was determined from the extracts by automated ion exchange chromatographic amino acid analysis (10). High-resolution ${ }^{1} \mathrm{H}$ NMR at $500 \mathrm{MHz}$ of extracts to which a known concentration of trimethylsilylpropionate had been added allowed separate measurement of brain phe and another phenyl-containing compound, as well as their total ([phenyl]). Serum [phe $]_{\text {aaa }}$ was determined by chromatographic amino acid analysis (10).

\section{RESULTS}

Figure 1 shows a ${ }^{1} \mathrm{H}$ brain spectrum obtained from a normal rabbit in vivo, with the excitation maximum of the binomial pulse sequence set at $7.37 \mathrm{ppm}$. The upfield region $(0-4 \mathrm{ppm})$ contains the familiar resonances found in brain spectra obtained in vivo with longer spin echo delays, including those from trimethylamines at $3.26 \mathrm{ppm}$, total creatine at $3.03 \mathrm{ppm}$, and $\mathrm{N}-$ acetyl aspartate at $2.02 \mathrm{ppm}$. The major resonances of glutamate, aspartate, and glutamine are in the region between 2.3 and 2.6 
ppm, labeled "amino acids." The downfield region (6-9 ppm) in the control spectrum consistently contains six resonances that have relatively short $T_{2} S$ and have not been conclusively assigned.

Figure 2 compares part of the downfield region $(6.5-8.5 \mathrm{ppm})$ of spectra obtained from the brains of normal and hyper-phe rabbits in vivo. In the hyper-phe animal (A10 in Table 1), there is an additional peak at $7.37 \mathrm{ppm}$, corresponding to the aromatic protons of phenyl. In this animail, serum [phe] aaa was $5.1 \mathrm{mM}$, and subsequent analysis of brain samples by amino acid analyzer and ${ }^{1} \mathrm{H}$ NMR yielded values for brain [phe] aaa $_{\text {and [phenyl] of }}$ 2.3 and $1.6 \mathrm{mM}$, respectively. Brain [phenyl] estimated from in vivo spectra was $2.0 \mathrm{mM}$.

High field ${ }^{1} \mathrm{H}$ NMR analysis of the brain extracts revealed the presence in most brains of an additional aromatic multiplet displaced slightly upfield of the phe multiplet (Fig. 3). The amount of this extra compound varied (Table 1). This additional set of peaks would not be resolved from the phe resonance in situ. Pending firm assignment, we designate their source as "compound X." This complex multiplet is observed as a single broad resonance in in vivo spectra due to the higher field strength used in the in vitro NMR analysis (11.8 Tesla versus 4.7 Tesla), broadening of each peak in the multiplet due to greater magnetic field inhomogeneity present under in vivo conditions, and variation in the intensity and location of these peaks that is dependent on field strength.

Table 1 summarizes the data obtained from 11-hyper-phe animals. As shown in Figure 4, the in situ determination of brain [phenyl] was in excellent agreement with brain [phe] aaa determined from postmortem brain samples. This conclusion was borne out by statistical comparison using the method of Bland and Altman (14), which uses differences between paired samples and is therefore appropriate when the original data are not normally distributed. The test found no significant difference at the $95 \%$ confidence limit between brain [phenyl] determined by in situ ${ }^{1} \mathrm{H}$ NMR and amino acid analysis. Comparison of in situ and in vitro ${ }^{1} \mathrm{H} N \mathrm{NMR}$ data showed excellent agreement when the latter included compound $\mathrm{X}$. This suggests that both phe and compound $\mathrm{X}$ were measured by both the in situ NMR method ([phenyl]) and the amino acid analyzer method (brain [phe $]_{\mathrm{aaa}}$ ).

Figure 5 shows the correlation between brain [phenyl] measured in situ and the corresponding serum [phe $]_{\text {aaa }}$. It is clear that serum [phe] $]_{\text {aaa }}$ was a poor predictor of brain [phenyl], being greater in all cases, and five times greater in the most extreme case. Since compound $X$ was present in all but one animal and comprised up to $74 \%$ of brain [phenyl], the discrepancy between brain [phe] and serum [phe] $]_{\text {aaa }}$ may have been even greater than the discrepancy in Figure 5.

\section{DISCUSSION}

Our study demonstrates that brain [phenyl] can be measured in vivo by ${ }^{1} \mathrm{H}$ NMR when serum [phe $]_{\mathrm{aaa}}$ is in the range usually found in hyper-phe animals and humans. The results also highlight two other features of the hyper-phe state, both possibly of importance for understanding of human PKU.

Brain [phenyl] may be quite different from serum [phe] $]_{\text {aaa. }}$ Under the conditions of our experiments, phe clearly did not achieve equal concentrations on both sides of the blood-brain barrier (Figure 5). Phe must either cross that barrier very slowly, or be maintained at a reduced concentration in the brain by some active process. These possibilities could be investigated by animal experiments in which the relative rates of clearance of phe from blood and brain were measured, the latter by ${ }^{1} \mathrm{H}$ NMR. Similar experiments should be possible in human PKU patients.

Compound $X$. The identity of compound $\mathrm{X}$ is presently unknown. It is not the inhibitor $\alpha$-methyl-phe, which has a characteristic methyl resonance at $1.56 \mathrm{ppm}$ in the ${ }^{1} \mathrm{H}$ NMR spectrum. It could be a metabolite of phe that is also present in human PKU and other chronic hyper-phe states, or merely an artifact of this particular animal model-another aromatic com- pound that accumulates as a consequence of inhibition by $\alpha$ methyl phe of enzymes other than phe-4-monooxygenase. Our data do not reveal whether it is produced in the brain, or produced elsewhere and taken up by the brain. However, preliminary studies based on analysis of the multiplet structure of the $\mathrm{X}$ resonance indicate that it may be $\gamma$-glutamyl-phe. Formation of this dipeptide would be favored in brain, where glutamate concentrations are normally $8-10 \mathrm{mM}$, and would be consistent with reports suggesting that some metabolites are removed from the brain by first conjugating them with glutamate (13). If it is present in the brains of patients with hyper-phe, it may play a part in the neurologic pathophysiology of those conditions. However, it might well be present in brain but not blood, making its role difficult to assess. As our study shows, its NMR signal is not separable from that of phe in vivo. If it is shed into the cerebrospinal fluid, it could be detected in lumbar puncture samples by high-resolution NMR (15).

NMR spectrometers in which humans can be studied at 4 Tesla are now available (16), and the techniques described here, when combined with quite rudimentary spatial localization schemes, should permit spectra similar to those shown here to be obtained in humans with hyper-phe. Although the chemical shift dispersion is smaller at the fields of 1.5-2.1 Tesla more generally available for human ${ }^{1} \mathrm{H}$ NMR spectroscopy, it should still be possible to detect a peak at $7.37 \mathrm{ppm}$ attributable to elevated brain [phenyl] in human phenylketonurics who do not follow a low-phe diet, since the effective line width of the downfield multiplet of phe should become narrower as the couplings become more AB in character (7). Such measurement capability would permit direct examination of the relationship between brain [phenyl] and the severity of neurophyschologic impairment found in some hyper-phe patients when they are off the low-phe diet. It would also allow monitoring of brain [phenyl] during treatments such as neutral amino acid supplements (17, 18) or somatic cell gene therapy (19). Direct measurement of brain [phenyl] may be particularly important for such evaluations in view of the pronounced discrepancy between serum [phe] $]_{\text {aaa }}$ and brain [phenyl] in this study. If brain [phenyl] were shown to persist for days after serum [phe] aaa reached normal levels, medical capability to monitor therapy would be extended. If the ratio of brain [phenyl] to serum [phe] $]_{\text {aaa }}$ was found to vary widely among individual PKU patients, new understanding of the pathophysiology of the disease might be achieved.

Acknowledgments. The authors thank Lisa Pleban and Peter Demou for essential technical assistance.

\section{REFERENCES}

1. Rosenberg, LE, Scriver CR 1980 Disorders of amino-acid metabolism. In: Bondy PK, Rosenberg LE (eds) Metabolic Control and Disease. WB Saunders, Philadelphia, pp 583-776

2. Scriver CR, Kaufman S, Woo SLC 1988 Mendelian hyperphenylalaninemia. Annu Rev Genet 22:301-321

3. Waisbren SE, Mahon BE, Schnell RR, Levy HL 1987 Predictors of intelligence quotient and intelligence quotient change in persons treated for phenylketonuria early in life. Pediatrics 79:351-355

4. Seashore MR, Friedman E, Novelly RA, Bapat V 1985 Loss of intellectual function in children with phenylketonuria after relaxation of dietary phenylalanine restriction. Pediatrics 75:226-232

5. Prichard JW, Shulman RG 1986 NMR spectoscopy of brain metabolism in vivo. Ann Rev Neurosci 9:61-85

6. Bottomley $\mathrm{P} 1989$ Human in vivo NMR spectroscopy in diagnostic medicine: clinical tool or research probe? Radiology 170:1-15

7. Gadian DG, Proctor E, Williams SR, Cady EB, Gardiner RM 1986 Neurometabolic effects of an inborn error of amino acid metabolism demonstrated in vivo by proton NMR. Magn Reson Med 3:150-156

8. Hore PJ 1983 Solvent suppression in fourier transform nuclear magnetic resonance. J Magn Reson 55:283-300

9. Bodenhausen G, Freeman R, Turner DL 1977 Suppression of artifacts in twodimensional J spectroscopy. J Magn Reson 27:511-514

10. Bachmann C, Colombo JP 1983 Increased tryptophan uptake into the brain in hyperammonemia. Life Sci 33:2417-2424 
11. Behar KL, den Hollander JA, Petroff OAC, Hetherington HP, Prichard JW, Shulman RG 1985 The effect of hypoglycemic encephalopathy upon amino acids, high energy phosphates, and $\mathrm{pHi}$ in the rat brain in vivo: detection by sequential ${ }^{1} \mathrm{H}$ and ${ }^{31} \mathrm{P}$ NMR spectroscopy. J Neurochem 44:1045-1055

12. Petroff OAC, Ogino T, Alger JR 1988 High-resolution proton magnetic resonance spectroscopy of rabbit brain: Regional metabolite levels and postmortem changes. J Neurochem 51:163-171

13. Mcllwain H, Bachelard HS 1985 Biochemistry of the Central Nervous System Churchill Livingstone, Edinborough, pp 170-171

14. Bland JM, Altman DG 1986 Statistical methods for assessing agreement between two methods of clinical measurement. Lancet 1:307-310

15. Petroff OAC, Yu RK, Ogino T 1986 High-resolution proton magnetic resonance analysis of human cerebrospinal fluid. J Neurochem 47:1270-1276
16. Bomsdorf H, Helzel T, Kunz D, Roschmann P, Tschendel O, Wieland J 1988 Spectroscopy and imaging with a 4 tesla whole body MR system. NMR in Biomedicine 1:151-158

17. Lou HC, Lykkelund S, Gerdes AM, Udesen H, Bruhn P 1987 Increased vigilance and dopamine synthesis by large doses of tyrosine or phenylalanine restriction in phenylketonuria. Acta Paediatr Scand 76:560-565

18. Ledley FD, Grenett HE, McGinnis-Shelnutt M, Woo SLC 1986 Retroviralmediated gene transfer of human phenylalanine hydroxylase into NIH-3T3 and hepatoma cells. Proc Natl Acad Sci USA 83:409-413

19. Ledley FD, Grenett HE, DiLella AG, Kwok SCM, Woo SLC 1985 Gene transfer and expression of human phenylalanine hydroxylase. Science 225:77-79 